\title{
Homology-based annotation of non-coding RNAs in the genomes of Schistosoma mansoni and Schistosoma japonicum Claudia S Copeland ${ }^{1,2}$, Manja Marz ${ }^{1}$, Dominic Rose ${ }^{1}$, Jana Hertel ${ }^{1}$, Paul J Brindley ${ }^{2}$, Clara Bermudez Santana ${ }^{1,8}$, Stephanie Kehr ${ }^{1}$, Camille Stephan-Otto Attolini ${ }^{3}$ and Peter F Stadler*1,4,5,6,7
}

Address: ${ }^{1}$ Bioinformatics Group, Department of Computer Science and Interdisciplinary Center for Bioinformatics, University of Leipzig, Härtelstraße 16-18, D-04107 Leipzig, Germany, ${ }^{2}$ Department of Microbiology, Immunology \& Tropical Medicine, George Washington University Medical Center, 2300 I Street, NW, Washington, DC 20037, USA, ${ }^{3}$ Memorial Sloan-Kettering Cancer Center, Computational Biology Department, 1275 York Avenue, Box \# 460, New York, NY 10065, USA, ${ }^{4}$ Max Planck Institute for Mathematics in the Sciences, Inselstrasse 22, D-04103 Leipzig, Germany, ${ }^{5}$ Fraunhofer Institute for Cell Therapy and Immunology, Perlickstraße 1, D-04103 Leipzig, Germany, ${ }^{6}$ Santa Fe Institute, 1399 Hyde Park Rd, Santa Fe, NM 87501, USA, ${ }^{7}$ Institute for Theoretical Chemistry, University of Vienna, Währingerstraße 17, A-1090 Wien, Austria and ${ }^{8}$ Department of Biology, National University of Colombia, Carrera 45 No. 26-85, Bogotá, D.C., Colombia

Email: Claudia S Copeland - cclaudia@bioinf.uni-leipzig.de; Manja Marz - manja@bioinf.uni-leipzig.de; Dominic Rose - dominic@bioinf.unileipzig.de; Jana Hertel - jana@bioinf.uni-leipzig.de; Paul J Brindley - mtmpjb@gwumc.edu; Clara Bermudez Santana - clara@bioinf.unileipzig.de; Stephanie Kehr - steffi@bioinf.uni-leipzig.de; Camille Stephan-Otto Attolini - camille@bioinf.uni-leipzig.de;

Peter F Stadler* - studla@bioinf.uni-leipzig.de

* Corresponding author

Published: 8 October 2009

BMC Genomics 2009, 10:464 doi:10.1/86/|47|-2/64-10-464
Received: 27 May 2009

Accepted: 8 October 2009

This article is available from: http://www.biomedcentral.com/I47I-2/64//0/464

(c) 2009 Copeland et al; licensee BioMed Central Ltd.

This is an Open Access article distributed under the terms of the Creative Commons Attribution License (http://creativecommons.org/licenses/by/2.0), which permits unrestricted use, distribution, and reproduction in any medium, provided the original work is properly cited.

\begin{abstract}
Background: Schistosomes are trematode parasites of the phylum Platyhelminthes. They are considered the most important of the human helminth parasites in terms of morbidity and mortality. Draft genome sequences are now available for Schistosoma mansoni and Schistosoma japonicum. Non-coding RNA (ncRNA) plays a crucial role in gene expression regulation, cellular function and defense, homeostasis, and pathogenesis. The genome-wide annotation of ncRNAs is a non-trivial task unless well-annotated genomes of closely related species are already available.

Results: A homology search for structured ncRNA in the genome of $S$. mansoni resulted in 23 types of ncRNAs with conserved primary and secondary structure. Among these, we identified rRNA, snRNA, SL RNA, SRP, tRNAs and RNase P, and also possibly MRP and 7SK RNAs. In addition, we confirmed five miRNAs that have recently been reported in $S$. japonicum and found two additional homologs of known miRNAs. The tRNA complement of S. mansoni is comparable to that of the free-living planarian Schmidtea mediterranea, although for some amino acids differences of more than a factor of two are observed: Leu, Ser, and His are overrepresented, while Cys, Meth, and Ile are underrepresented in S. mansoni. On the other hand, the number of tRNAs in the genome of $S$. japonicum is reduced by more than a factor of four. Both schistosomes have a complete set of minor spliceosomal snRNAs. Several ncRNAs that are expected to exist in the $S$. mansoni genome were not found, among them the telomerase RNA, vault RNAs, and $Y$ RNAs.

Conclusion: The ncRNA sequences and structures presented here represent the most complete dataset of ncRNA from any lophotrochozoan reported so far. This data set provides an important reference for further analysis of the genomes of schistosomes and indeed eukaryotic genomes at large.
\end{abstract}




\section{Background}

Non-coding RNA (ncRNA) plays a crucial role in gene expression regulation, cellular function and defense, and disease. Indeed, in higher eukaryotes, most of the genomic DNA sequence encodes non-protein-coding transcripts [1]. In contrast to protein-coding mRNAs, ncRNAs do not form a homogeneous class. The best-characterized subclasses form stable basepairing patterns (secondary structures) that are crucial for their function. This group includes the well-known tRNAs, catalytically active RNAs such as rRNA, snRNAs, RNase P RNA, and other ribozymes, and regulatory RNAs such as microRNAs and spliceosomal RNAs that direct protein complexes to specific RNA targets. Much less is known about long mRNAlike ncRNAs, which are typically poorly conserved at the level of both sequence and structure.

Most non-vertebrate genome projects have put little emphasis on a comprehensive annotation of ncRNAs. Indeed, most non-coding RNAs, with the notable exception of tRNAs and rRNAs, are difficult or impossible to detect with BLAST in phylogenetically distant organisms. Hence, ncRNA annotation is not part of generic genome annotation pipelines. Dedicated computational searches for particular ncRNAs, for example, RNase P and MRP [2,3], 7SK RNAs [4,5], or telomerase RNA [6,7], are veritable research projects in their own right. Despite best efforts, ncRNAs across the animal phylogeny remain to a large extent uncharted territory.

The main difficulty with ncRNA annotation is poor sequence conservation and indel patterns that often correspond to large additional "expansion domains". In many cases, the secondary structure is much better conserved than the primary sequence, providing a means of confirming candidate ncRNAs even in cases where sequence conservation is confined to a few characteristic motifs. Secondary structure conservation can also be utilized to detect homologs of some ncRNAs based on characteristic combinations of sequence and structure motifs using special software tools designed for this purpose.

In [8] we described a protocol for a more detailed homology-based ncRNA annotation than what can be achieved with currently available automatic pipelines. Here, we apply this scheme to the genome of $S$. mansoni, and by comparison with the newly sequenced $S$. japonicum genome, identify ncRNAs in both of these clinically important schistosomes.

Schistosomes belong to an early-diverging group within the Digenea, but are clearly themselves highly derived [911]. The flatworms are a long-branch group, suggesting rapid mutation rates (see [12]).
Schistosome genomes are comparatively large, estimated to be over 350 megabase pairs, and perhaps as high as 400 megabase pairs, for the haploid genome of $S$. mansoni and S. japonicum [13-15]. The other major schistosome species parasitizing humans probably have a genome of similar size, based on the similarity in appearance of their karyotypes [16]. These large sizes may be characteristic of platyhelminth genomes in general: the genome of Schmidtea mediterranea is even larger, with the current genome sequencing project reporting a size of $\sim 480$ million base pairs [17] http://genome.wustl.edu/genomes/view/schmi dtea mediterranea/.

Genome sequencing of the seven autosomes and the pair of sex chromosomes of $S$. mansoni with about $8 \times$ coverage has lead to a genome assembly comprising 5,745 scaffolds (> $2 \mathrm{~kb}$ ) covering $363 \mathrm{Mb}[13,14,18]$. Similarly, shotgun sequencing of $S$. japonicum with coverage of $5.4 \times$ decoded $397 \mathrm{Mb}$ of sequence [15]. These form about 25,000 scaffolds. Albeit both genome projects did not lead to complete finished genomes, we therefore know at least 90$95 \%$ of the genomic DNA sequences of S. japonicum and S. mansoni, respectively.

The protein-coding portion of the Schistosoma genomes have received much attention in recent years. Published work includes transcriptome databases for both S. japonicum [19] and S. mansoni [20], microarray-based expression analysis [21], characterization of promoters [22,23], and physical mapping and annotation of protein-coding genes from both the $S$. mansoni and $S$. japonicum genome projects [18]. Recently, a systematic annotation of protein-coding genes in S. japonicum was reported [24]. In contrast to other, better-understood, parasites such as Plasmodium [25], however, not much is known about the non-coding RNA complement of schistosomes. Only the spliced leader RNA (SL RNA) of S. mansoni [26], the hammer-head ribozymes encoded by the SINE-like retrotransposons $\mathrm{Sm}-\alpha$ and $\mathrm{Sj}-\alpha[27,28]$, and secondary structure elements in the LTR retrotransposon Boudicca [29] have received closer attention. Ribosomal RNA sequences have been available mostly for phylogenetic purposes [30], and tRNAs have been studied to a limited degree [31].

The wealth of available ESTs, in principle, provides a valuable resource for ncRNA detection. Since mostly poly-A ESTs have been generated, it is not surprising that most ESTs have been attributed to protein-coding genes [32]. The large evolutionary distance, with $55 \%$ of the genes without homologs outside the genus [13,18], makes it hard or even impossible to reliably distinguish ESTs of putative mRNA-like ncRNAs from non-coding portions of protein-coding transcripts. 
In this contribution we therefore focus on a comprehensive overview of the evolutionary conserved non-coding RNAs in the genomes of S. mansoni and S. japonicum. We discuss representatives of 23 types of ncRNAs that were detected based on both sequence and secondary structure homology.

\section{Results and discussion}

Structure and homology-based searches of the schistosome genomes revealed ncRNAs from 23 different RNA categories. Table 1 lists these functional ncRNA categories, the number of predicted genes in each category, and references associated with each RNA type. Supplementary fasta files containing the ncRNA genes, bed files with the genome annotation, and stockholm-format alignment files can be accessed at http://www.bioinf.uni-leip zig.de/Publications/SUPPLEMENTS/08-014.

\section{Transfer RNAs}

Candidate tRNAs were predicted with tRNAscan-SE in the genomes of $S$. mansoni, S. japonicum and S. mediterranea (a free-living platyhelminth, used for comparison). After removal of transposable element sequences (see below), tRNAscan-SE predicted a total of $713 \mathrm{tRNAs}$ for S. mansoni and 739 for $S$. mediterranea, while 154 tRNAs were found in the $S$. japonicum sequences. These included tRNAs encoding the standard 20 amino acids of the traditional genetic code, selenocysteine encoding tRNAs (tRNAsec) [33] and possible suppressor tRNAs [34] in all three genomes. The tRNAsec from schistosomes has been characterized, and is similar in both size and structure to tRNAsec from other eukaryotes [35].

The tRNA complements of the three platyhelminth genomes are compared in detail in Figure 1. The amino

Table I: Summary of homology-based RNA annotations from the sequenced genomes of S. mansoni and S. japonicum.

\begin{tabular}{|c|c|c|c|c|}
\hline RNA class & Functional Category & S. man. & S. jap. & Related reference(s) \\
\hline 7SK & Transcription regulation & $(1)$ & 0 & This study \\
\hline Hammerhead ribozymes & Self-cleaving & $>38,000$ & $>5,000$ & {$[27]$} \\
\hline miRNA & Translation control & 8 & 7 & [109], this study \\
\hline potassium channel motif & RNA editing & 9 & 3 & [65] \\
\hline RNase MRP & Mitochondrial replication, rRNA processing & $(1)$ & ( & This study \\
\hline RNase $P$ & tRNA processing & 1 & 1 & This study \\
\hline rRNA-operon & Polypeptide synthesis & $80-105$ & $50-280$ & [39], this study \\
\hline $5 S$ rRNA & Polypeptide synthesis & 21 & $1-13$ & This study \\
\hline SL RNA & Trans-splicing & $6-48$ & $1-9$ & [26], this study \\
\hline SnoRNA U3 & Nucleolar rRNA processing & I & I & This study \\
\hline SRP & Protein transportation & 12 & $4+1$ & This study \\
\hline tRNA & Polypeptide synthesis & 663 & 154 & This study \\
\hline UI & Splicing & $3-34$ & $2-6$ & [44], this study \\
\hline U2 & Splicing & $3-15$ & $1-63$ & {$[44]$, this study } \\
\hline U4 & Splicing & $1-19$ & $1-6$ & [44], this study \\
\hline U5 & Splicing & $2-9$ & $\mathrm{I}-24$ & [44], this study \\
\hline U6 & Splicing & $9-55$ & $2-12$ & [44], this study \\
\hline UII & Splicing & 1 & 1 & This study \\
\hline $\mathrm{U} 12$ & Splicing & $\mathrm{I}-2$ & $0-1$ & [44], this study \\
\hline U4atac & Splicing & I & 1 & This study \\
\hline U6atac & Splicing & 1 & 1 & This study \\
\hline U7 & Histone maturation & 0 & $(2)$ & This study \\
\hline
\end{tabular}

Where are range of numbers is given, it remains uncertain whether multiple copies in the genomic DNA are true copies of the gene or assembly artifacts. 

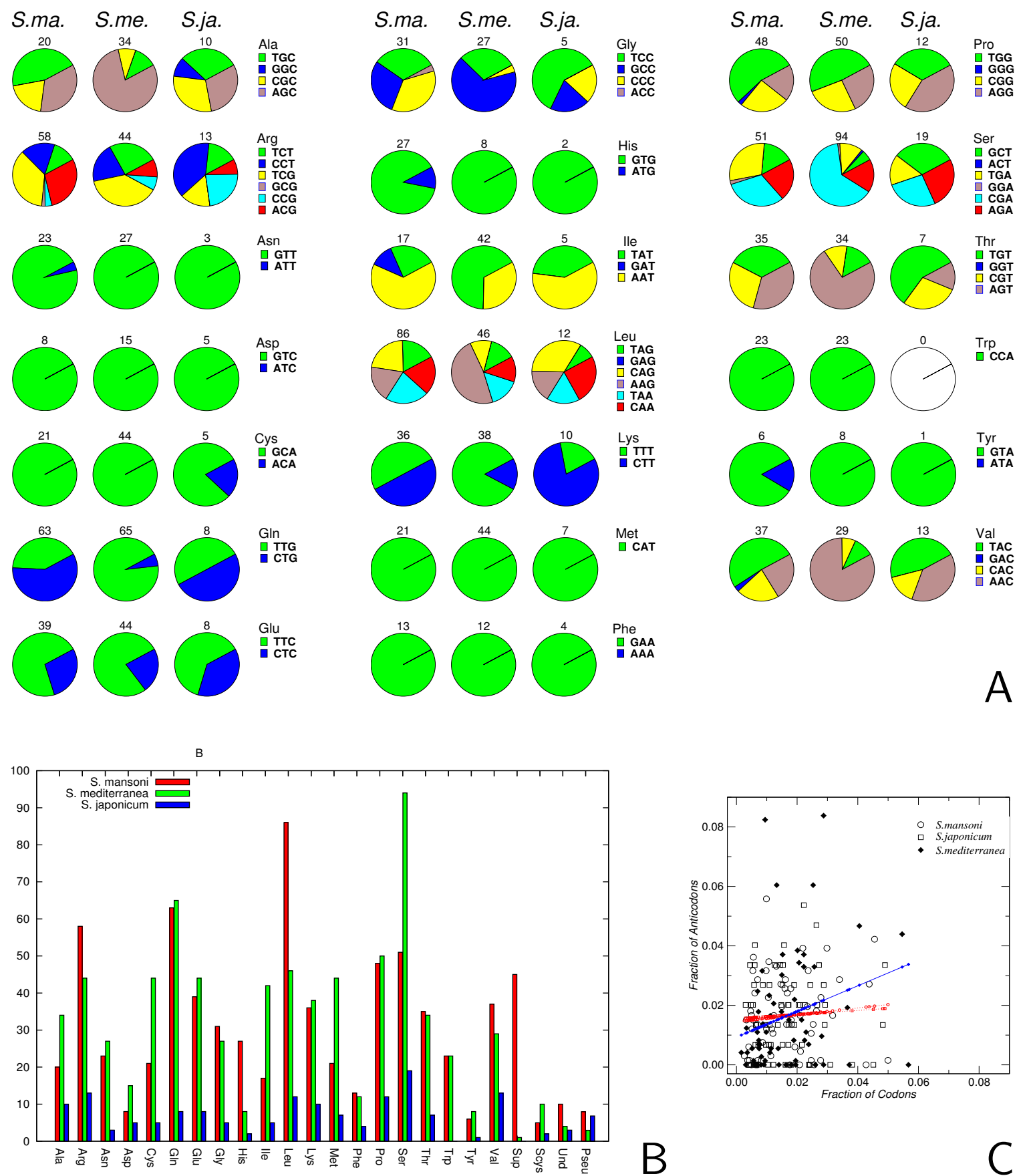

Figure I

Comparison of the tRNA complement of Schistosoma mansoni, Schistosoma japonicum, and Schmidtea mediterranea. A: Comparison of anti-codon distributions for the 20 amino acids. Numbers below each pie-chart are the total number of tRNA genes coding the corresponding amino acid. Left columns: S. mansoni; middle columns: $S$. mediterranea; right columns: S. japonicum. B: Number of tRNAs encoding a particular amino acid. red: S. mansoni, blue: S. japonicum, green: S. mediterranea. Abbreviations: Sup: putative suppressor tRNAs (CTA, TTA); Scys: Selenocysteine tRNAs (TCA); Pseu: predicted pseudogenes; Und: tRNA predictions with uncertain anticodon; likely these are also tRNA pseudogenes. The Gln-tRNA derived repeat family (see text) is not included in these data. $\mathbf{C}$ : Comparison of codon usage and anti-codon abundance. No significant correlation is observed for the two schistosomes. For S. mediterranea there is a weak, but statistically significant, positive correlation: $t \approx 2.0$ 
acids are represented in approximately equal numbers in S. mansoni and Schmidtea. Nevertheless, there are several notable deviations. S. mansoni contains many more leucine ( 86 vs. 46 ) and histidine ( 27 vs. 8 ) tRNAs, while serine (51 vs. 94), cysteine ( 21 vs. 44 ), methionine (21 vs. 44 ), and isoleucine (17 vs. 42 ) are underrepresented. In addition, there are several substantial differences in codon usage. In most cases, $S$. mansoni has a more diverse repertoire of tRNAs: tRNA-Asn-ATT, tRNA-Arg-CGC, tRNA-HisATG, tRNA-Ile-GAT, tRNA-Pro-GGG, tRNA-Tyr-ATA, tRNA-Val-GAC are missing in Schmidtea. Only tRNA-SerACT is present in Schmidtea but absent in Schistosoma. The tRNA complement of $S$. japonicum, on the other hand, differs strongly from its two relatives. Not only is the number of tRNAs decreased by more than a factor of four, $S$. japonicum also prefers anticodons that are absent or rare in its relatives, such as tRNA-Ala-GGC, tRNA-Cys-ACA, and LysCTT. On the other hand, no tRNA-Trp was found. Since the UGG codon is present in many open reading frames we interpret this as a problem with the incompleteness of the genome assembly rather than a genuine gene loss. The reduction in the number of tRNAs is also evident by comparing the number of tRNAs with introns: 27 in S. mansoni versus 5 in $S$. japonicum.

It has been shown recently that changes in codon usage, even while coding the same protein sequences, can severely attenuate the virulence of viral pathogens [36] by "de-optimizing" translational efficiency. This observation leads us to speculate that the greater diversity of the tRNA repertoire could be related to the selection pressures of the parasitic life-style of $S$. mansoni. The effect is not straightforward, however, because there is no significant correlation of tRNA copy numbers with the overall codon usage in both S. mansoni and S. japonicum, Figure 1C. In contrast, a weak but statistically significant correlation can be observed in Schmidtea mediterranea. It would be interesting, therefore, to investigate in detail whether there are differences in codon usage of proteins that are highly expressed in different stages of $S$. mansoni's life cycle, and whether the relative expression levels of tRNAs are under stage-specific regulation.

The most striking result of the tRNAscan-SE analysis was the initial finding of 1,135 glutamine tRNAs (GlntRNAs) in S. mansoni in contrast to the 8 Gln-tRNAs in $S$. japonicum and 65 in $S$. mediterranea. Nearly all of these (1,098 in S. mansoni) were tRNA-Gln-TTG. In addition, an extreme number of 1,824 tRNA-pseudogenes in $S$. mansoni (vs. 951 in S. japonicum and 19 in S. mediterranea) was predicted. Of these, 1,270 were also homologous to tRNA-Gln-TTG. These two groups of tRNA-Gln-TTGderived genes (those predicted to be pseudogenes and those predicted to be functional tRNAs) totaled 2,368. These high numbers suggest a tRNA-derived mobile genetic element. We therefore ran the 2,368 S. mansoni tRNA-Gln-TTG genes through the RepeatMasker program [37]. Almost all of them $(2,342)$ were classified as SINE elements. Further BLAST analysis revealed that these elements are similar to members of the $\mathrm{Sm}-\alpha$ family of $S$. mansoni SINE elements [38]. Removal of these SINE-like elements yielded a total of 63 predicted glutamine-encoding tRNAs in S. mansoni. About 650 of 951 pseudogenes in $S$. japonicum derived from tRNA-Pro-CGG.

Homology-based analysis yielded similar, though somewhat less sensitive, results to those of tRNAscan-SE. For instance, a BLAST search in $S$. mansoni with Rfam ' s tRNA consensus yielded 617 predicted tRNAs compared to the 663 predictions made by tRNAscan-SE.

\section{Ribosomal RNAs}

As usual in eukaryotes, the $18 \mathrm{~S}, 5.8 \mathrm{~S}$, and $28 \mathrm{~S}$ genes are produced by RNA polymerase I from a tandemly repeated polycistronic transcript, the ribosomal RNA operon. The S. mansoni genome contains about $90-100$ copies $[39,40]$ which are nearly identical at sequence level, because they are subject to concerted evolution [41]. The repetitive structure of the rRNA operons causes substantial problems for genome assembly software [42]. In order to obtain a conservative estimate of the copy number, we retained only partial operon sequences that contained at least two of the three adjacent rRNA genes. We found 48 loci containing parts of $18 \mathrm{~S}, 5.8 \mathrm{~S}$, and $28 \mathrm{~S}$ genes, 32 loci covering $18 \mathrm{~S}$ and $5.8 \mathrm{~S}$ rRNA, and 57 loci covering $5.8 \mathrm{~S}$ and 28S rRNAs [see Additional file 1 - Figures S1 and S2]. Adding the copy numbers, we have not fewer than 80 copies (based on linked 18S rRNAs) and no more than 137 copies (based on linked 5.8S rRNA). The latter is probably an overestimate due to the possibility that the $5.8 \mathrm{~S}$ rRNA may be contained in two scaffolds. The copy number of rRNA operons is thus consistent with the estimate of 90100 from hybridization analysis [39]. An analogous analysis of the current $S$. japonicum assembly yields less accurate results. Due to the many short fragments, we obtained 90 copies; the true number may lie between 50 and 280, however.

The 5S rRNA is a polymerase III transcript that has not been studied in schistosomes so far. We found 21 copies of the $118 \mathrm{nt}$ long $5 \mathrm{~S}$ rRNA in S. mansoni, compared with 13 copies in $S$. japonicum. Four of the 21 copies are located within a 3,000 nt cluster on Scaffold010519.

\section{Spliceosomal RNAs and Spliced Leader RNA}

Spliceosomes, the molecular machines responsible for most splicing reactions in eukaryotic cells, are ribonucleoprotein complexes similar to ribosomes [43]. The major spliceosome, which cleaves GT-AG introns, includes the five snRNAs U1, U2, U4, U5, and U6. In the 
S. mansoni genome, all of them are multicopy genes. By homology search we found 34 U1, 15 U2, 19 U4, 9 U5, and $55 \mathrm{U} 6$ sequences in the genome assembly. Interpreting all sequences that are identical in short flanking regions as the same, we would retain only $3 \mathrm{U} 1,3 \mathrm{U} 2,1$ U4, 2 U5, and nine U6 genes [44]. The true copy number in the $S$. mansoni genome is most likely somewhere between these upper and lower bounds. For $S$. japonicum, the corresponding numbers are U1: 2-6, U2: 1-63 U2, U4: 1-6 U4, U5: 1-24, and U6: 2-12. Due to the more fragmented genome assembly we expect the true numbers to be closer to the lower bounds. Secondary structures for these candidates are similar to those of typical snRNAs, Figure 2.

A second, much less frequent, minor spliceosome is responsible for the processing of atypical AT-AC introns. It shares only the U5 snRNA with the major spliceosome. The other four RNA components are replaced by variants called U11, U12, U4atac, and U6atac [45]. The minorspliceosomal snRNAs are typically much less conserved than the RNA components of the major spliceosome [44]. It was not surprising, therefore, that these RNAs were detectable only by means of GotohScan[8] but not with the much less sensitive BLAST searches. Although U4atac and U6atac are quite diverged compared to known homologs, they can be recognized unambiguously based on both secondary structure and conserved sequence motifs. Furthermore, the U4atac and U6atac sequences can interact to form the functionally necessary duplex structure shown in Figure 2. As in many other species, there is only a single copy of each of the minor spliceosomal snRNAs in both of the schistosome genomes, Tab. 1. An analysis of promoter sequences showed that the putative snRNA promoter motifs in S. mansoni are highly derived. Only one of the two U12 genes exhibited a clearly visible snRNA-like promoter organization.

The Spliced Leader (SL) RNA is one of the very few previously characterized ncRNAs from S. mansoni [26]. The 90 nt SL RNA, which was found in a 595 nt tandemly repeated fragment (accession number M34074), contains the $36 \mathrm{nt}$ leader sequence at its 5 ' end which is transferred in the trans-splicing reaction to the 5 ' termini of mature mRNAs. Using blastn, we identified 54 SL RNA genes. These candidates, along with 100 nt flanking sequence, were aligned using ClustalX, revealing 6 sequences with aberrant flanking regions, which we suspect to be pseudogenic. The remaining sequences are 43 identical copies and 5 distinct sequence variants. A secondary structure analysis corroborates the model of [26], according to which the S. mansoni SL RNA has only two loops, with an
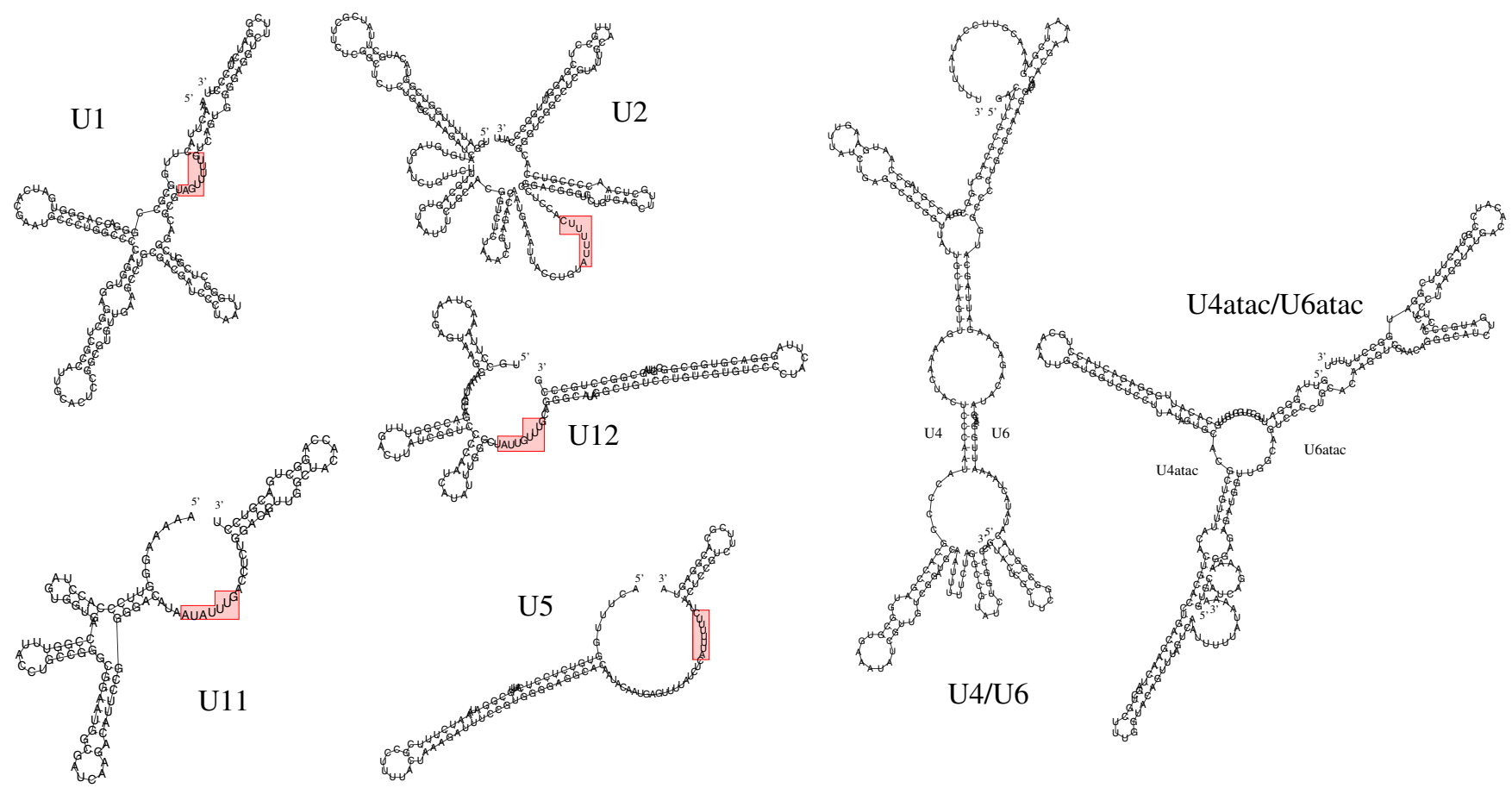

Figure 2

Secondary structures of the nine snRNAs and the interaction complexes of U4/U6 and U4atac/U6atac, respectively, in S. mansoni. Structure prediction was performed by RNAfold, RNAal i fold and for U4/U6 and U4atac/U6atac by RNAcofold from the RNA Vienna Package $[96,108]$. Boxes indicate Sm binding sites. Additional details on sequences, structures, and alignments are available at the supplementary material. 
unpaired Sm binding site [see Additional File 1 - Figure S3]. This coincides with the SL RNA structure of Rotifera [46], but is in contrast to the SL RNAs in most other groups of eukaryotes, which exhibit single or triple stemloop structures [47]. A blastn-search against S. mansoni EST data confirms that the 5' part of the SL is indeed transspliced to mRNAs. Several nearly identical SL RNA homologs are found in S. japonicum.

\section{SRP RNA and Ribonuclease P RNA}

Signal recognition particle (SRP) RNA, also known as 7SL RNA, is part of the signal recognition particle, a ribonucleoprotein that directs packaged proteins to their appropriate locations in the endoplasmic reticulum. Although one of the protein subunits of this ribonucleoprotein was cloned in 1995 [48], little is known about the other subunits or the RNA component in S. mansoni. We found eight probable candidates for the SRP RNA, with one almost canonical sequence [see Additional file 1 - Figure S4], and four possible candidates with point mutations which may influence their function.

The RNA component of Ribonuclease P (RNase P) is the catalytically active part of this enzyme that is required for the processing of tRNA precursors $[49,50]$. We found one classic RNase P RNA in the S. mansoni genome using both Gotohscan and rnabob with the eukaryotic ("nuclear") Rfam consensus sequence for RNase P as search sequence.

\section{MicroRNAs}

MicroRNAs are small RNAs that are processed from hairpin-like precursors, see e.g. [51]. They are involved in post-transcriptional regulation of mRNA molecules. So far, no microRNAs have been verified experimentally in $S$. mansoni. The presence of four protein-coding genes encoding crucial components of the microRNA processing machinery (Dicer, Argonaut, Drosha, and Pasha/ DGCR8) [52,53], and the presence of Argonaut-like genes in both S. japonicum [54] and S. mansoni (detected by tblastn in EST data, see Supplemental Data online), strongly suggests that schistosomes have a functional microRNA system. Indeed, most recently five miRNAs were found by direct cloning in $S$. japonicum that are also conserved in S. mansoni [55]: let-7, mir-71, bantam, mir125 , and a single schistosome-specific microRNA. These sequences, including the precursor hairpins, are well conserved in $S$. japonicum. On the other hand, the microRNA precursor sequences of both schistosomes are quite diverged from the consensus of the homologous genes in Bilateria.

Using bioinformatics (see methods) we were able to find only one further miRNA candidate in S. mansoni, mir-124, that is also conserved in $S$. japonicum. In insects, this miRNA is clustered with mir-287. The distance of both
miRNAs is approximately $8 \mathrm{~kb}$ in Drosophilids. We found an uncertain mir-287 candidate in S. mansoni, however, on a different scaffold than mir-124. Although this sequence nicely folds into a single stem-loop structure, it is conserved only antisense to the annotated mature sequence in insects (see, Figure 3). This S. mansoni mir-287 candidate does not seem to be conserved in S. japonicum.

In [56], 71 microRNAs are described for the distantly related trematode Schmidtea mediterranea, and additional ones are announced in a recent study focussing on piRNAs [57]. The overwhelming majority, 54, were reported to be members of 29 widely conserved metazoan microRNA families, although in some cases even the mature miRNA sequence is quite diverged. Therefore, we regard several family assignments as tentative at best. Of those $29 \mathrm{miR}$ NAs, we found mir-124 only. However, the schistosome sequences are more related to the other bilaterian mir-124 homologs than to those of $S$. mediterranea. Out of the remaining 54 miRNAs that were annotated in $S$. mediterranea we found that mir-749 is also conserved in the two schistosome species. Here, the sequences show a common consensus sequence and secondary structure in their precursors (see Figure 3).

The small number of recognizable microRNAs in schistosomes is in strong contrast to the extensive microRNA complement in S. mediterranea, indicating massive loss of microRNAs relative to the planarian ancestor. This may be a consequence of the parasitic lifestyle of the schistosomes.

\section{Small Nucleolar RNAs}

Small nucleolar RNAs play essential roles in the processing and modification of rRNAs in the nucleolus [58,59]. Both major classes, the box H/ACA and the box C/D snoRNAs are relatively poorly conserved at the sequence level and hence are difficult to detect in genomic sequences. This has also been observed in a recent ncRNA annotation project of the Trichoplax adhaerens genome [8]. The bestconserved snoRNA is the atypical U3 snoRNA, which is essential for processing of the $18 \mathrm{~S}$ rRNA transcript into mature $18 \mathrm{~S}$ rRNA [60]. In the current assembly of the $S$. mansoni genome we found six U3 loci, but they are also identical in the flanking sequences, suggesting that in fact there is only a single U3 gene. No unambiguous homologue was detected for any of the other known snoRNAs.

A de novo search for snoRNAs (see methods for details) resulted in 2,610 promising candidates $(1,654$ box $C / D$ and 956 box H/ACA), see Supplemental Data online. All these predictions exhibit highly conserved sequence boxes as well as the typical secondary features of box C/D and box H/ACA snoRNAs, respectively. 


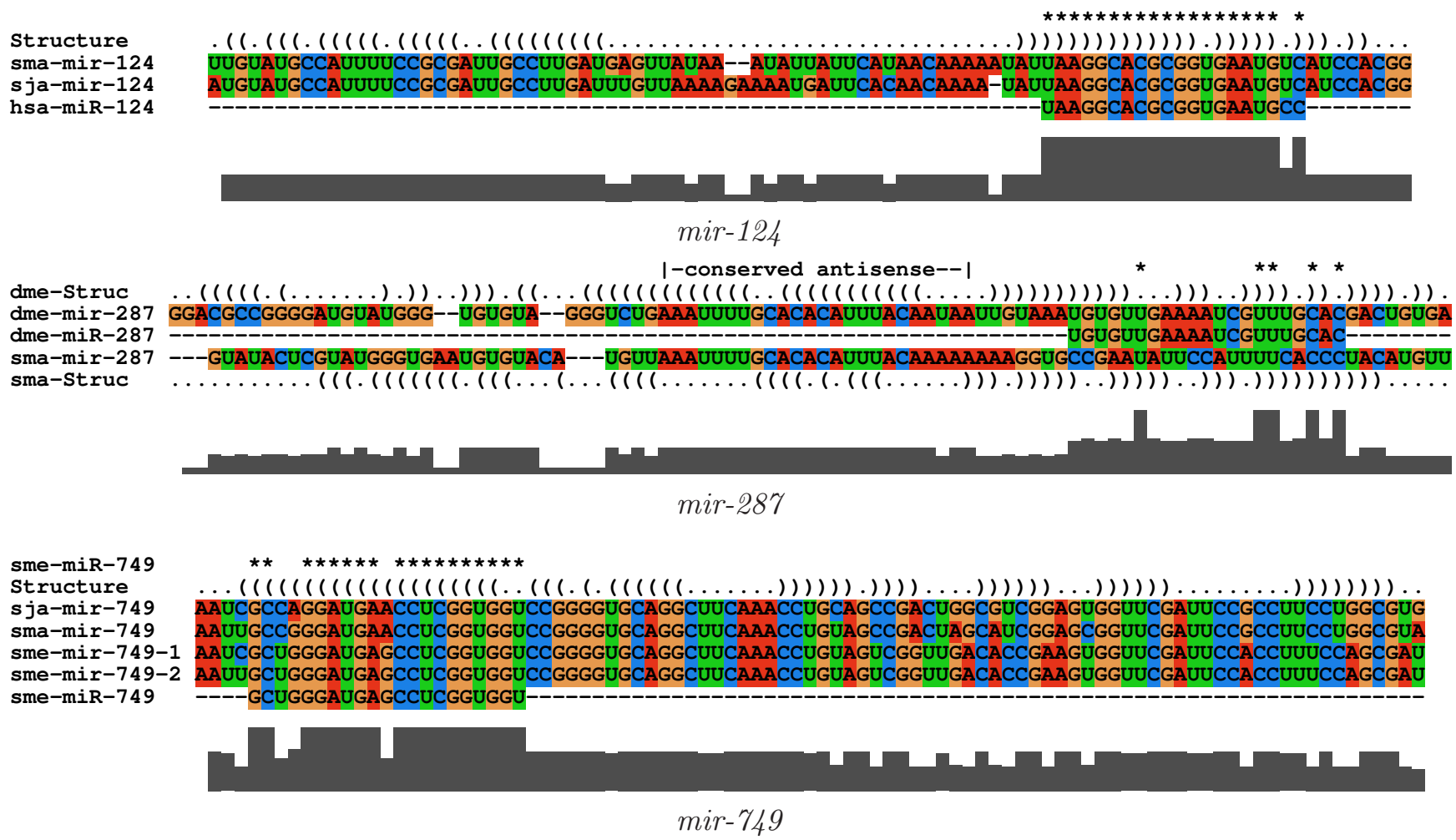

Figure 3

Multiple sequence alignments of the pre-miRNAs that were computationally found in S. mansoni. For mir- 124 and mir-749 the sequences share a common consensus structure. The uncertain mir-287 candidate clusters together with mir- 124 in insect genomes. However, though it also exhibits a single stem-loop structure, it is different from that of insects. Here the sequence is only conserved at the antisense region of the annotated mature miRNA.

A comparison of the predicted snoRNAs with the entries in the Rfam[61] and NONCODE[62] databases returned only 47 hits that match to several other RNAs like tRNAs, parts of the rRNA operon, snRNAs, mRNAlike genes and a few of our candidates map to the hammerhead ribozyme. These sequences are likely false positives and have been removed from the candidate list. The number of predicted candidates is much larger than the number of snoRNAs reported in other organisms; for instance [59] lists 456 for the human genome. Although we most likely do not yet know the full snoRNA complement of eukaryotic genomes, we have to expect that a large fraction of prediction will turn out to be false positives.
We therefore analysed the conservation of the candidates in $S$. japonicum and focussed on the snoRNA candidates with targets in the $18 \mathrm{~S}, 28 \mathrm{~S}$ and/or $5.8 \mathrm{~S}$ ribosomal RNA. While targets are predicted for more than half of the candidates, see Table 2, the numbers are drastically reduced when conservation of the candidates in S. japonicum is required. Note, furthermore, that the fraction of conserved candidates is strongly enriched among those with ribosomal RNA targets, indicating that these sets are likely to contain a sizeable fraction of true positives. This filtering step leaves us with 227 box C/D and 352 box H/ACA snoRNA candidates. While still high, these numbers fall into the expected range for a metazaon snoRNA complement.

Table 2: Conservation and target prediction of snoRNA candidates.

\begin{tabular}{|c|c|c|c|c|c|c|}
\hline \multirow[t]{2}{*}{ snoReporttargets } & \multicolumn{3}{|c|}{ Box C/D (snoscan) } & \multicolumn{3}{|c|}{ Box H/ACA (RNAsnoop) } \\
\hline & $\geq \mathbf{2}$ & I & 0 & $\geq \mathbf{2}$ & 1 & 0 \\
\hline predicted in S. mansoni & 926 & 110 & 613 & 284 & 495 & 177 \\
\hline conserved in S. japonicum & 200 & 27 & 83 & 149 & 203 & 62 \\
\hline
\end{tabular}

Only ribosomal RNAs were searched for putative target sites. 
We remark, finally, that five of the snoRNA candidates (three box C/D and two box H/ACA) are also conserved in Schmidtea mediterranea.

\section{Other RNA motifs}

Two examples of relatively well-known schistosome noncoding RNAs are the hammerhead ribozyme motifs within the Sm- $\alpha$ and Sj- $\alpha$ SINE-like elements $[27,28]$. A blastn search of the hammerhead ribozyme motif from the Rfam database resulted in $\sim 38,500$ candidates for $S$. mansoni in contrast to $\sim 5,000$ candidates for $S$. japonicum. While high, this number is not surprising considering the generally high copy number of SINE elements; previously, the copy number for Sm- $\alpha$ elements in the $S$. mansoni genome was estimated to exceed 10,000 [27]. The highly conserved potassium channel RNA editing signal $[63,64]$ is another structured RNA element that was described previously [65]. We found nine copies of this hairpin structure in the $S$. mansoni genome assembly and three in $S$. japonicum.

\section{Uncertain and missing candidates}

Both the MRP RNA $[2,3,66]$ and the 7SK RNA $[4,5,67]$ have highly variable, rapidly evolving sequences that make them difficult or impossible to detect in invertebrate genomes. Their ancient evolutionary origin and their extremely conserved molecular house-keeping functions make it more than likely that they are present in the schistosome genomes as well. In both cases, we have not been able to identify unambiguous homologs. There are, however, plausible candidates. We briefly describe them in the following paragraphs since they may warrant further attention and may be a useful starting point for subsequent experimental studies, as exemplified by the history of discovery of the snRNA in Giardia intestinalis [68-70].

MRP RNA has multiple functions, among them mitochondrial RNA processing and nucleolar pre-rRNA processing. The $S$. mansoni MRP candidate fits the general secondary structure model of metazoan MRP RNAs $[2,3,66]$ and analysis with RNAduplex shows that the candidate contains a pseudoknot which exhibited striking sequence identity with known MRPs. The locus is wellconserved in S. japonicum. On the other hand, stems 1 and 12 were divergent compared to known MRPs, and stem 19 also fails to display clear similarities with known MRPs. Although quite likely a true MRP homolog, we therefore consider this sequence only tentative.

7SK RNA is a general transcriptional regulator, repressing transcript elongation through inhibition of transcription elongation factor PTEFb and also suppresses the deaminase activity of APOBEC3C [71]. The S. mansoni 7SK candidate has a $5^{\prime}$ stem similar to that described in other invertebrates [5], and parts of the middle of the sequence are also recognizable. There is, furthermore, a homolo- gous locus in the genome of S. japonicum. However, the 3' stem (which was followed by a poly-T terminator) was not conserved. In addition, a large sequence deletion was evident.

Three major classes of ncRNAs were expected, but not found, in the S. mansoni genome. As in all other invertebrates genomes, no candidate sequence was found for a telomerase RNA. S. mansoni almost certainly has a canonical telomerase holoenzyme, since it encodes telomerase proteins (Smp_066300 and Smp_066290) and has the same telomeric repeat sequences as many other metazoan animals [72]. Telomerase RNAs are notoriously difficult to find, as they are highly divergent among different species, varying in both size and sequence composition $[7,73]$. Vault RNAs are known in all major deuterostome lineages [74], and homologs were recently also described in two lophotrochozoan lineages [75]. Since S. mansoni has a homolog of the major vault protein (Smp_006740) we would also expect a corresponding RNA component to be present. So far, Y RNAs have been found only in vertebrates $[76,77]$ and in nematodes $[78,79]$, although the Ro RNP, that they are associated with, seems to be present in most or even all eukaryotes.

\section{Conclusion}

We have described here a detailed annotation of "housekeeping" ncRNAs in the genomes of the parasitic platyhelminth Schistosoma mansoni and Schistosoma japonicum. Limited to the best conserved structured RNAs, our work nevertheless uncovered important genomic features such as the existence of a SINE family specific to Schistosoma mansoni, which is derived from tRNA-Gln-TTG. Our data furthermore establish the presence of a minor spliceosome in schistosomes and confirms spliced leader transsplicing. With a coverage of at least $90-95 \%$ of the genomic DNA, missing data are not a significant problem. The fragmented genome assemblies, however, preclude accurate counts of the multi-copy genes.

Platyhelminths are known to be a fast-evolving phylum [80]. It is not surprising therefore that in particular the small ncRNAs are hard or impossible to detect by simple homology search tools such as blastn. Even specialized tools have been successful in identifying only the better conserved genes such as tRNA, microRNAs, RNase P RNA, SRP RNA. The notoriously poorly conserved families, such as snoRNAs, telomerase RNA, or vault RNAs, mostly escaped detection.

The description of several novel, and in many cases quite derived, schistosome ncRNAs contributes significantly to the understanding of the evolution of the corresponding RNA families. The schistosome ncRNA sequences, furthermore, are an important input to subsequent homology search projects, since they allow the construction of 
improved descriptors for sequence/structure-based search algorithms. Last but not least, the ncRNA annotation tracks are an important contribution to the genome-wide annotation datasets of both $S$. mansoni and S. japonicum. It not only contributes the protein-based annotation but also helps to identify annotation errors, e.g. cases where putative proteins are annotated that overlap rRNA operons or other ncRNAs.

The house-keeping ncRNAs considered in this study are almost certainly only the proverbial tip of the platyhelminth ncRNAs iceberg. The discovery of a large number of mRNA-like ncRNAs (mlncRNAs) in many eukaryotes (compiled e.g. in the RNAdb[81] and reviewed e.g. in [1]), and in particular in many other invertebrate species (nematodes [82], insects [83,84]) suggests that similar transcripts will also be abundant in schistosomes. The abundant EST data for both schistosome species $[85,86]$ can provide a starting point e.g. for an analysis along the lines of [87]. Computational surveys, furthermore, have provided evidence for large numbers of RNAs with conserved secondary structures in other invertebrates [88-90]. The underlying methods, such as RNAz[91], are inherently comparative, presenting difficulties for application to schistosome genomes due to the large evolutionary distance between schistosome and non-schistosome genomes. This is also the case for a recent approach to identify mRNA-like non-coding RNAs with very low levels of sequence conservation based on their intron structure [92]. A deeper understanding of the non-coding transcriptome of schistosomes will therefore have to rely primarily on experimental approaches, either by means of tiling arrays or by means of high throughput transcriptome sequencing.

\section{Methods \\ tRNA annotation}

We used tRNAscan-SE[93] with default parameters to annotate putative tRNA genes. As additional confirmation, the genome sequence was searched using tRNA consensus sequences from the Rfam database [61]. In order to obtain suitable data for comparison, the genome of the free-living platyhelminth Schmidtea mediterranea [17] was searched alongside that of S. mansoni and S. japonicum.

\section{microRNA annotation}

We followed the general protocol outlined in [8] to identify miRNA precursors, using all metazoan miRNAs listed in miRBase [94] [Release 11.0, http://micro rna.sanger.ac.uk/sequences/]. The initial search was conducted by blastn with $E<0.01$ with the mature and mature* miRNAs as query sequences. The resulting candidates were then extended to the length of the precursor sequence of the search query and aligned to the precursors using ClustalW[95]. Secondary structures were predicted using RNAfold[96] for single sequences and
RNAalifold[97] for alignments. Candidates that did not fold into miRNA-like hairpin structures were discarded. The remaining sequences were then examined by eye to see if the mature miRNA was well-positioned in the stem portion of each putative precursor sequence. In addition, we used the final candidates to search the S. japonicum and $S$. mediterranea genomes to examine whether these sequences are conserved in Schistosoma and/or Platyhelminthes.

\section{snoRNA annotation}

We compared all the known human and yeast snoRNAs that are annotated in the snoRNAbase[98] to the $S$. mansoni genome using BLAST[99] and GotohScan[8]. The search for novel snoRNA candidates was performed only on sequences that were not annotated as protein-coding or another ncRNA in the current $S$. mansoni assembly. The SnoReport program [100] was used to identify putative box C/D and box H/ACA snoRNAs on both strands. Only the best predictions, i.e., those that show highly conserved boxes and canonical structural motifs, were kept for further analysis. The remaining candidates are further analysed for possible target interactions with ribosomal RNAs using snoscan[101] for box C/D and RNAsnoop[102] for box H/ACA snoRNA candidates. In addition, the sequences were checked for conservation in $S$. japonicum and $S$. mediterranea using BLAST. To estimate the number of false predictions we compared the candidate snoRNAs with common ncRNA databases, in particular Rfam[61] and NONCODE[62]. All sequences that match a nonsnoRNA ncRNA were discarded.

\section{Other RNA families}

For other families, we employed the following five steps:

(a) For candidate sequences of ribosomal RNAs, spliceosomal RNAs, the spliced leader (SL) and the SRP RNA, we performed BLAST searches with $E<0.001$ using the known ncRNA genes from the NCBI and Rfam databases. For the snRNA set, see [44]. For 7SL RNA we used X04249, for $5 \mathrm{~S}$ and $5.8 \mathrm{~S}$ rRNAs we used the complete set of Rfam entries, for the SSU and LSU rRNAs, we used $Z 11976$ and NR_003287, respectively. The SL RNAs were searched using SL RNA entries from Rfam and the sequences reported in [26]. For more diverged genes such as minor snRNAs, RNase MRP, 7SK, and RNase $P$, we used Gotohscan[8], an implementation of a full dynamic programming alignment with affine gap costs. In cases where no good candidates were found we also employed descriptor-based search tools such as rnabob http:// selab.janelia.org/software.html.

(b) In a second step, known and predicted sequences were aligned using Clustalw[95] and visualized with Clus talX[103]. To identify functional secondary structure, RNAfold, RNAalifold, and RNAcofold[104] were 
used. Combined primary and secondary structures were visualized using stockholm-format alignment files in the emacs editor utilizing ralee mode [105]. Alignments are provided at the Supplemental Data online.

(c) Putatively functional sequences were distinguished from likely pseudogenes by analysis of flanking genomic sequence. To this end, the flanking sequences of snRNA and SL RNA copies were extracted and analyzed for conserved sequence elements using MEME[106]. Only snRNAs with plausible promoter regions were reported.

(d) Additional consistency checks were employed for individual RNA families, including phylogenetic analysis by neighbor-joining [107] to check that candidate sequences fall at phylogenetically reasonable positions relative to previously known homologs. For RNase MRP RNA candidates, RNAduplex http://www.tbi. uni vie.ac.at/RNA/RNAduplex.html was used to find the pseudoknot structure. In order to confirm that the SL RNA candidate is indeed trans-spliced to mRNA transcripts, we searched the FAPESP Genoma Schistosoma mansoni website for ESTs including fragments of the predicted SL RNA. We found 52 ESTs with blastnE $<0.001$ that span the predicted region of the SL RNA (nt 8-38), indicating that this RNA does indeed function as a spliced leader.

(e) Accepted candidate sequences were used as BLAST queries against the $S$. mansoni genome to determine their copy number in the genome assembly.

\section{Additional Data Online}

The website http://www.bioinf.uni-leipzig.de/Publica tions/SUPPLEMENTS/08-014 provides extensive machine readable information, including sequence files, alignments, and genomic coordinates.

\section{Authors' contributions}

CSC, PB, and PFS designed the study. CSC, MM, DR, JH, CBS, SK, CSA, and PFS performed the computational analyses. CSC wrote the first draft of the manuscript. All authors contributed to the final assessment of the data as well as the writing of the final version of the manuscript. CSC, MM, DR, JH should be considered as joint first authors.

\section{Additional material}

\section{Additional file 1}

Supplemental figures and captions. contains supplemental Figures S1 S4 mentioned in the main text.

Click here for file

[http://www.biomedcentral.com/content/supplementary/14712164-10-464-S1.PDF]

\section{Acknowledgements}

This work was supported in part by the European Union through grants in the 6th and 7th Framework Programe of the European Union (projects EMBIO, SYNLET, and EDEN), the Deutsche Forschungsgemeinschaft and the auspices of SPP SPP-I 174 "Deep Metazoan Phylogeny", the Freistaat Sachsen, and the DAAD-AleCol program.

\section{References}

I. Amaral PP, Dinger ME, Mercer TR, Mattick JS: The eukaryotic genome as an RNA machine. Science 2008, 31 9:1787-1789.

2. Piccinelli P, Rosenblad MA, Samuelsson T: Identification and analysis of ribonuclease $P$ and MRP RNA in a broad range of eukaryotes. Nucleic Acids Res 2005, 33:4485-4495.

3. Woodhams MD, Stadler PF, Penny D, Collins LJ: RNAse MRP and the RNA Processing Cascade in the Eu-karyotic Ancestor. BMC Evol Biol 2007, 7:SI3.

4. Gruber AR, Koper-Emde D, Marz M, Tafer H, Bernhart S, Obernosterer G, Mosig A, Hofacker IL, Stadler PF, Benecke BJ: Invertebrate 7SK snRNAs. J Mol Evol 2008, 1 07-I I 5:66.

5. Gruber A, Kilgus C, Mosig A, Hofacker IL, Hennig W, Stadler PF: Arthropod 7SK RNA. Mol Biol Evol 2008, 1923-1930:25.

6. Chen JL, Blasco MA, Greider CW: Secondary Structure of Vertebrate telomerase RNA. Cell 2000, 100:503-5।4.

7. Xie M, Mosig A, Qi X, Li Y, Stadler PF, Chen JJL: Size Variation and Structural Conservation of Vertebrate Telomerase RNA. J Biol Chem 2008, 283:2049-2059.

8. Hertel J, de Jong D, Marz M, Rose D, Tafer H, Tanzer A, Schierwater B, Stadler PF: Non-Coding RNA Annotation of the Genome of Trichoplax adhaerens. Nucleic Acids Res 2009, 37:1602-1615.

9. Blair D, Davis GM, Wu B: Evolutionary relationships between trematodes and snails emphasizing schistosomes and paragonimids. Parasitology 200I, I 23(Suppl):S229-S243.

10. Brant SV, Loker ES: Can specialized pathogens colonize distantly related hosts? Schistosome evolution as a case study. PLoS Pathog 2005, I:167-169.

II. Webster BL, Southgate VR, Littlewood DTJ: A revision of the interrelationships of Schistosoma including the recently described Schistosoma guineensis. Int J Parasitol 2006, 36:947-955.

12. Jiménez-Guri E, Philippe H, Okamura B, Holland PWH: Buddenbrockia is a cnidarian worm. Science 2007, 317:116-118.

13. Wilson RA, Ashton PD, Braschi S, Dillon GP, Berriman M, Ivens A: 'Oming in on schistosomes: prospects and limitations for post-genomics. Trends Parasitol 2007, 23: 14-20.

14. Berriman M, Haas BJ, LoVerde PT, Wilson RA, Dillon GP, Cerqueira GC, Mashiyama ST, Al-Lazikani B, Andrade LF, Ashton PD, Aslett MA, Bartholomeu DC, Blandin G, Caffrey CR, Coghlan A, Coulson R, Day TA, Delcher A, DeMarco R, Djikeng A, Eyre T, Gamble JA, Ghedin E, Gu Y, Hertz-Fowler C, Hirai H, Hirai Y, Houston R, Ivens A, Johnston DA, Lacerda D, Macedo CD, McVeigh P, Ning Z, Oliveira G, Overington JP, Parkhill J, Pertea M, Pierce RJ, Protasio AV, Quail M, Rajandream MA, Rogers J, Sajid M, Salzberg SL, Stanke M, Tivey AR, White O, Williams DL, Wortman J, Wu W, Zamanian M, Zerlotini A, FraserLiggett CM, Barrell BG, El-Sayed NM: The genome of the blood fluke Schistosoma mansoni. Nature 2009, 460:352-358.

15. Schistosoma japonicum Genome Sequencing and Functional Analysis Consortium: The Schistosoma japonicum genome reveals features of host-parasite interplay. Nature 2009, 460:345-35I.

16. Hirai $H$, Taguchi $T$, Saitoh $Y$, Kawanaka $M$, Sugiyama $H$, Habe $S$, Okamoto M, Hirata M, Shimada M, Tiu WU, Lai K, Upatham ES, Agatsuma T: Chromosomal differentiation of the Schistosoma japonicum complex. Int J Parasitol 2000, 30:44I-452.

17. Robb SMC, Ross E, Alvarado AS: SmedGD: the Schmidtea mediterranea genome database. Nucleic Acids Res 2008, 36:D599-D606.

18. Haas BJ, Berriman M, Hirai H, Cerqueira GG, Loverde PT, El-Sayed NM: Schistosoma mansoni genome: closing in on a final gene set. Exp Parasitol 2007, I 1 7:225-228.

19. Hu W, Yan Q, Shen DK, Liu F, Zhu ZD, Song HD, Xu XR, Wang ZJ, Rong YP, Zeng LC, Wu J, Zhang X, Wang J], Xu XN, Wang SY, Fu G, Zhang XL, Wang ZQ, Brindley PJ, McManus DP, Xue CL, Feng Z, Chen Z, Han ZG: Evolutionary and biomedical implications of a Schistosoma japonicum complementary DNA resource. Nat Genet 2003, 35:|39-| 47. 
20. Verjovski-Almeida S, R D, Martins EA, Guimarães PE, Ojopi EP, Paquola AC, Piazza JP, Nishiyama MY Jr, Kitajima JP, Adamson RE Ashton PD, Bonaldo MF, Coulson PS, Dillon GP, Farias LP, Gregorio SP, Ho PL, Leite RA, Malaquias LC, Marques RC, Miyasato PA, Nascimento AL, Ohlweiler FP, Reis EM, Ribeiro MA, Sá RG, Stukart GC, Soares MB, Gargioni C, Kawano T, Rodrigues V, Madeira AM, Wilson RA, Menck CF, Setubal JC, Leite LC, Dias-Neto E: Transcriptome analysis of the acoelomate human parasite Schistosoma mansoni. Nat Genet 2003, 35: I48-I57.

21. Verjovski-Almeida S, Venancio TM, Oliveira KC, Almeida GT, DeMarco R: Use of a 44k oligoarray to explore the transcriptome of Schistosoma mansoni adult worms. Exp Parasitol 2007, I | 7:236-245.

22. Schulmeister A, Heyers O, Morales ME, Brindley PJ, Lucius R, Meusel $\mathrm{G}$, Kalinna $\mathrm{BH}$ : Organization and functional analysis of the Schistosoma mansoni cathepsin D-like aspartic protease gene promoter. Biochim Biophys Acta 2005, I 727:27-34.

23. Copeland CS, Mann VH, Brindley PJ: Both sense and antisense strands of the LTR of the Schistosoma mansoni Pao-like retrotransposon Sinbad drive luciferase expression. Mol Genet Genomics 2007, 277:I6I-I70.

24. Brejová B, Vinaz T, Chen Y, Wang S, Zhoa G, Brown DG, Li M, Zhou Y: Finding genes in Schistosoma japonicum: annotating nove genomes with help of extrinsic evidence. Nucleic Acids Res 2009 37:e52.

25. Mourier T, Carret C, Kyes S, Christodoulou Z, Gardner PP, Jeffares DC, Pinches R, Barrell B, Berriman M, Griffiths-Jones S, Ivens A, Newbold C, Pain A: Genome-wide discovery and verification of novel structured RNAs in Plasmodium falciparum. Genome Res 2008, I 8:281-292.

26. Rajkovic A, Davis RE, Simonsen JN, Rottman FM: A spliced leader is present on a subset of mRNAs from the human parasite Schistosoma mansoni. Proc Natl Acad Sci USA 1990, 87:8879-8883.

27. Ferbeyre G, Smith JM, Cedergren R: Schistosome satellite DNA encodes active hammerhead ribozymes. Mol Cell Biol 1998, I 8:3880-3888.

28. Laha T, McManus DP, Loukas A, Brindley PJ: Sj $\alpha$ elements, short interspersed element-like retroposons bearing a hammerhead ribozyme motif from the genome of the oriental blood fluke Schistosoma japonicum. Biochim Biophys Acta 2000 , 1 492:477-482

29. Copeland CS, Heyers O, Kalinna BH, Bachmair A, Stadler PF, Hofacker IL, Brindley PJ: Structural and evolutionary analysis of the transcribed sequence of Boudicca, a Schistosoma mansoni retrotransposon. Gene 2004, 329:103-II4.

30. Rollinson D, Kaukas A, Johnston DA, Simpson AJ, Tanaka M: Some molecular insights into schistosome evolution. Int J Parasito 1997, 27: I I-28.

31. Littlewood DT, Lockyer AE, Webster BL, Johnston DA, Le TH: The complete mitochondrial genomes of Schistosoma haematobium and Schistosoma spindale and the evolutionary history of mitochondrial genome changes among parasitic flatworms. Mol Phylogenet Evol 2006, 39:452-467.

32. DeMarco R, Verjovski-Almeida S: Expressed Sequence Tags (ESTs) and Gene Discovery: Schistosoma mansoni. Bioinformat ics in Tropical Disease Research: A Practical and Case-Study Approach 2008:B06 [http://www.ncbi.nlm.nih.gov/bookshelf/br.fcgi?book=bio info]. Bethesda, MD: National Library of Medicine

33. Sheppard K, Akochy PM, Söll D: Assays for transfer RNA dependent amino acid biosynthesis. Methods 2008, 44: I39- I 45.

34. Ambrogelly A, Palioura S, Söll D: Natural expansion of the genetic code. Nat Chem Biol 2007, 3:29-35

35. Hubert N, Walczak R, Sturchler C, Myslinski E, Schuster C, Westhof E, Carbon P, Krol A: RNAs mediating cotrans-lational insertion of selenocysteine in eukaryotic selenoproteins. Biochimie 1996 , 78:590-596.

36. Coleman JR, Papamichail D, Skiena S, Futcher B, Wimmer E, Mueller $\mathrm{S}$ : Virus attenuation by genome-scale changes in codon pair bias. Science 2008, 320:1784-1787.

37. Smit AFA, Hubley R, Green P: RepeatMasker. [Version, open3.2.5 [RMLib: 200806 I I]. [http://www.repeatmasker.org/].

38. Spotila LD, Hirai H, Rekosh DM, Lo Verde PT: A retroposon-like short repetitive DNA element in the genome of the human blood fluke, Schistosoma mansoni. Chromosoma 1989, 97:421-428.
39. Simpson AJ, Dame JB, Lewis FA, McCutchan TF: The arrangement of ribosomal RNA genes in Schistosoma mansoni. Identification of polymorphic structural variants. Eur J Biochem I984, | 39:4|-45.

40. van Keulen H, Loverde PT, Bobek LA, Rekosh DM: Organization of the ribosomal RNA genes in Schistosoma mansoni. Mol Biochem Parasitol 1985, I 5:215-230.

4I. Nei M, Rooney AP: Concerted and birth-and-death evolution of multigene families. Annu Rev Genet 2005, 39:। 2 I- I 52.

42. Scheibye-Alsing K, Hoffmann S, Frankel AM, Jensen P, Stadler PF, Mang Y, Tommerup N, Gilchrist MJ, Hillig ABN, Cirera S, Jørgensen $\mathrm{CB}$, Fredholm M, Gorodkin J: Sequence Assembly. Comp Biol Chem 2009, 33:121-136.

43. Staley JP, Woolford JL Jr: Assembly of ribosomes and spliceosomes: complex ribonucleoprotein machines. Curr Opin Cell Biol 2009, 2 I: 109- I I8.

44. Marz M, Kirsten T, Stadler PF: Evolution of Spliceosomal snRNA Genes in Metazoan Animals. J Mol Evol 2008, 67:594-607.

45. Kreivi JP, Lamond Al: RNA splicing: unexpected spliceosome diversity. Curr Biol 1996, 6:802-805

46. Pouchkina-Stantcheva NN, Tunnacliffe A: Spliced leader RNAmediated trans-splicing in phylum Rotifera. Mol Biol Evol 2005, 22: $1482-1489$.

47. Marz M, Vanzo N, Stadler PF: Carnival of SL RNAs: Structural variants and the possibility of a common origin. J Bioinf Comp Biol 2009 [http://www.bioinf.uni-leipzig.de/Publications/PREPRINTS/ 09-009.pdf].

48. McNair A, Zemzoumi K, Lütcke H, Guillerm C, Boitelle A, Capron A, Dissous $\mathrm{C}$ : Cloning of a signal-recognition-particle subunit of Schistosoma mansoni. Parasitol Res 1995, 81 : I75-I77.

49. Kirsebom LA: RNase P RNA mediated cleavage: substrate recognition and catalysis. Biochimie 2007, 89: I I83-I I 94.

50. Kikovska E, Svärd SG, Kirsebom LA: Eukaryotic RNase P RNA mediates cleavage in the absence of protein. Proc Natl Acad Sci USA 2007, 104:2062-2067.

5I. Williams AE: Functional aspects of animal microRNAs. Cell $\mathrm{Mol}$ Life Sci 2008, 65:545-562.

52. Krautz-Peterson G, Skelly PJ: Schistosoma mansoni: the dicer gene and its expression. Exp Parasitol 2008, I I 8: | 22- 128

53. Gomes MS, Cabral FJ, Jannotti-Passos LK, Carvalho O, Rodrigues V, Baba EH, Sá RG: Preliminary analysis of miRNA pathway in Schistosoma mansoni. Parasitol lnt 2009, 58:6I-68.

54. Liu F, Lu J, Hu W, Wang SY, Cui SJ, Chi M, Yan Q, Wang XR, Song HD, Xu XN, Wang J], Zhang XL, Zhang X, Wang ZQ, Xue CL, Brindley PJ, McManus DP, Yang PY, Feng Z, Chen Z, Han ZG: New perspectives on host-parasite interplay by comparative transcriptomic and proteomic analyses of Schistosoma japonicum. PLoS Pathog 2006, 2:e29.

55. Xue X, Sun J, Zhang Q, Wang Z, Huang Y, Pan W: Identification and characterization of novel microRNAs from Schistosoma japonicum. PLOS ONE 2008, 3:e4034.

56. Palakodeti D, Smielewska M, Graveley BR: MicroRNAs from the Planarian Schmidtea mediterranea: a model system for stem cell biology. RNA 2006, I 2: I 640-I 649.

57. Palakodeti D, Smielewska M, Lu YC, Yeo GW, Graveley BR: The PIWI proteins SMEDWI-2 and SMEDWI-3 are required for stem cell function and piRNA expression in planarians. RNA 2008, I4:II74-II86.

58. Matera AG, Terns R, Terns : Non-coding RNAs: lessons from the small nuclear and small nucleolar RNAs. Nat Rev Mol Cell Biol 2007, 8:209-220.

59. Dieci G, Preti M, Montanini B: Eukaryotic snoRNAs: A paradigm for gene expression flexibility. Genomics 2009, 94:83-88.

60. Lukowiak AA, Granneman S, Mattox SA, Speckmann WA, Jones K, Pluk WJ, Venrooij Hand, Terns RM, Terns MP: Interaction of the U3-55k protein with U3 snoRNA is mediated by the box B/C motif of $\mathbf{U} 3$ and the WD repeats of U3-55k. Nucleic Acids Res 2000, 28:3462-347I.

61. Griffiths-Jones S, Moxon S, Marshall M, Khanna A, Eddy SR, Bateman A: Rfam: annotating non-coding RNAs in complete genomes. Nucleic Acids Res 2005, 33:DI2I-DI 24.

62. Liu C, Bai B, Skogerbø G, Cai L, Deng W, Zhang Y, Bu DB, Zhao Y, Chen R: NONCODE: an integrated knowledge database of noncoding RNAs. Nucleic Acids Res 2005, 33:DI I2-D I I 5. 
63. Bhalla T, Rosenthal JJC, Holmgren M, Reenan R: Control of human potassium channel inactivation by editing of a small mRNA hairpin. Nature Struct Mol Biol 2004, I I:950-956.

64. Yang Y, Lv J, Gui B, Yin H, Wu X, Zhang Y, Jin Y: A-to-I RNA editing alters less-conserved residues of highly conserved coding regions: implications for dual functions in evolution. RNA 2008, I4:1516-1525.

65. Kim E, Day TA, Bennett JL, Pax RA: Cloning and functional expression of a Shaker-related voltage-gated potassium channel gene from Schistosoma mansoni (Trematoda: Digenea). Parasitology 1995, I I 0:171-180.

66. López MD, Rosenblad MA, Samuelsson T: Conserved and variable domains of RNase MRP RNA. RNA Biology 2009, 6:208-22I.

67. Marz M, Donath A, Verstraete N, Nguyen VT, Stadler PF, Bensaude $O$ : Evolution of 7SK RNA and its Protein Partners in Metazoa. Mol Biol Evol 2009 in press.

68. Collins LJ, Poole AM, Penny D: Using ancestral sequences to uncover potential gene homologues. Appl Bioin-formatics 2003, 2(Suppl 3):85-95

69. Chen XS, Rozhdestvensky TS, Collins LJ, Schmitz J, Penny D: Combined experimental and computational approach to identify non-protein-coding RNAs in the deep-branching eukaryote Giardia intestinalis. Nucleic Acids Res 2007, 35:4619-4628.

70. Chen XS, White WT, Collins LJ, Penny D: Computational identification of four spliceosomal snRNAs from the deep-branching eukaryote Giardia intestinalis. PLoS One 2008, 3(8):e3106.

71. Barrandon C, Spiluttini B, Bensaude O: Non-coding RNAs regulating the transcriptional machinery. Biol Cell 2008, 100:83-95.

72. Hirai H, LoVerde PT: Identification of the telomeres on Schistosoma mansoni chromosomes by FISH. J Parasitol I996, 82:511-512.

73. Theimer CA, Feigon J: Structure and function of telomerase RNA. Curr Opin Struct Biol 2006, I 6:307-3 I8.

74. Stadler PF, Chen JإL, Hackermüller J, Hoffmann S, Horn F, Khaitovich P, Kretzschmar AK, Mosig A, Prohaska SJ, Qi X, Schutt K, Ullmann K: Evolution of Vault RNAs. Mol Biol Evol 2009, 26:1975-1991.

75. Mosig A, Zhu L, Stadler PF: Strategies for Homology-Based ncRNA Gene Annotation. Brief Funct Genomics Proteomics 2009 in press.

76. Mosig A, Guofeng M, Stadler BMR, Stadler PF: Evolution of the Vertebrate Y RNA Cluster. Th Biosci 2007, I 26:9-I4.

77. Perreault J, Perreault JP, Boire G: Ro-associated Y RNAs in metazoans: evolution and diversification. Mol Biol Evol 2007 , 24:1678-1689

78. Van Horn DJ, Eisenberg D, O'Brien CA, Wolin SL: Caenorhabditis elegans embryos contain only one major species of Ro RNP. RNA 1995, I:293-303.

79. Boria I, Gruber AR, Tanzer A, Bernhart S, Lorenz R, Mueller MM Hofacker IL, Stadler PF: Nematode sbRNAs: homologs of vertebrate Y RNAs. Tech. Rep. BIOINF-09-020 2009 [http://www.bio inf.uni-leipzig.de/Publications/PREPRINTS/09-020.pdf]. Bioinformatics, University of Leipzig

80. Lartillot N, Brinkmann H, Philippe H: Suppression of long-branch attraction artefacts in the animal phylogeny using a site-heterogeneous model. BMC Evolutionary Biology 2007, 7:S4

8I. Pang KC, Stephen S, Dinger ME, Engström PG, Lenhard B, Mattick JS: RNAdb 2 . 0 -- an expanded database of mammalian non-coding RNAs. Nucleic Acids Res 2007, 35:DI78-DI82.

82. Shin $\mathrm{H}$, Hirst M, Bainbridge MN, Magrini V, Mardis E, Moerman DG Marra MA, Baillie DL, Jones SJ: Transcriptome analysis for Caenorhabditis elegans based on novel expressed sequence tags. BMC Biol 2008, 6:30.

83. Inagaki S, Numata K, Kondo T, Tomita M, Yasuda K, Kanai A, Kageyama $Y$ : Identification and expression analysis of putative mRNA-like non-coding RNA in Drosophila. Genes Cells 2005, I0:| | 63-1 I73.

84. Tupy JL, Bailey AM, Dailey G, Evans-Holm M, Siebel CW, Misra S, Celniker SE, Rubin GM: Identification of putative noncoding polyadenylated transcripts in Drosophila melanogaster. Proc Nat Acad Sci USA 2005, 1 02:5495-5500.

85. Zerlotini A, Heiges M, Wang $H$, Moraes RL, Dominitini AJ, Ruiz JC, Kissinger JC, Oliveira G: SchistoDB: a Schistosoma mansoni genome resource. Nucleic Acids Res 2009, 37:D579-D582.

86. Liu F, Chen P, Cui SJ, Wang ZQ, Han ZG: SjTPdb: integrated transcriptome and proteome database and analysis platform for Schistosoma japonicum. BMC Genomics 2008, 9:304.
87. Seemann SE, Gilchrist MJ, Hofacker IL, Stadler PF, Gorodkin J: Detection of RNA structures in porcine EST data and related mammals. BMC Genomics 2007, 8:316.

88. Missal K, Rose D, Stadler PF: Non-coding RNAs in Ciona intestinalis. Bioinformatics 2005, 2 I(S2):i77-i78.

89. Missal K, Zhu X, Rose D, Deng W, Skogerbø G, Chen R, Stadler PF: Prediction of Structured Non-Coding RNAs in the Genome of the Nematode Caenorhabitis elegans. J Exp Zool: Mol Dev Evol 2006, 306B:379-392

90. Rose DR, Hackermüller J, Washietl S, Findeiß S, Reiche K, Hertel J, Stadler PF, Prohaska SJ: Computational RNomics of Drosophilids. BMC Genomics 2007, 8:406.

91. Washietl S, Hofacker IL, Stadler PF: Fast and reliable prediction of noncoding RNAs. Proc Natl Acad Sci USA 2005, I 02:2454-2459.

92. Hiller M, Findeiß S, Lein S, Marz M, Nickel C, Rose D, Schulz C, Backofen R, Prohaska SJ, Reuter G, Stadler PF: Conserved Introns Reveal Novel Transcripts in Drosophila melanogaster. Genome Res 2009, 19:1289-1300.

93. Lowe T, Eddy S: tRNAscan-SE: a program for improved detection of transfer RNA genes in genomic sequence. Nucl Acids Res 1997, 25:955-964.

94. Griffiths-Jones S, Saini HK, van Dongen S, Enright AJ: miRBase: tools for microRNA genomics. Nucleic Acids Res 2008, 36:DI54-D|58.

95. Thompson JD, Higgs DG, Gibson TJ: CLUSTALW: improving the sensitivity of progressive multiple sequence alignment through sequence weighting, position specific gap penalties, and weight matrix choice. Nucl Acids Res 1994, 22:4673-4680.

96. Hofacker IL, Fontana W, Stadler PF, Bonhoeffer LS, Tacker M, Schuster P: Fast Folding and Comparison of RNA Secondary Structures. Monatsh Chem 1994, I 25:167-188.

97. Hofacker IL, Fekete M, Stadler PF: Secondary Structure Prediction for Aligned RNA Sequences. I Mol Biol 2002, 319:1059-1066.

98. Lestrade L, Weber MJ: snoRNA-LBME-db, a comprehensive database of human H/ACA and C/D box snoRNAs. Nucleic Acids Res 2006, 34:D158-D162.

99. Altschul SF, Gish W, Miller W, Myers EW, Lipman DJ: Basic local alignment search tool. J Mol Biol I990, 2I 5:403-4IO.

100. Hertel J, Hofacker IL, Stadler PF: snoReport: Computational identification of snoRNAs with unknown targets. Bioinformatics 2008, 24: I58-164.

I0I. Lowe TM, Eddy SR: A Computational Screen for Methylation Guide snoRNAs in Yeast. Science 1999, 283: I I68- I I7|

102. Tafer H, Kehr S, Hertel J, Stadler PF: RNAsnoop: Efficient target prediction for box H/ACA snoRNAs. Tech. Rep. BIOINF-09-025 2009 [http://www.bioinf.uni-leipzig.de/Publications/PREPRINTS/09. 025.pdf. Bioinformatics, University of Leipzig

103. Thompson JD, Gibson TJ, Plewniak F, Jeanmougin F, Higgins DG: The CLUSTAL $X$ windows interface: flexible strategies for multiple sequence alignment aided by quality analysis tools. Nucleic Acids Res 1997, 25:4876-4882

104. Bernhart SH, Tafer H, Mückstein U, Flamm C, Stadler PF, Hofacker IL: Partition Function and Base Pairing Probabilities of RNA Heterodimers. Algorithms Mol Biol 2006, I:3.

105. Griffiths-Jones S: RALEE --RNA ALignment editor in Emacs. Bioinformatics 2005, 21:257-259.

106. Bailey TL, Williams N, Misleh C, Li WW: MEME: discovering and analyzing DNA and protein sequence motifs. Nucleic Acids Res 2006, 34:W369-W373.

107. Saitou N, Nei M: The neighbor-joining method: a new method for reconstructing phylogenetic trees. Mol Biol Evol 1987, 4:406-425.

108. Hofacker IL: Vienna RNA secondary structure server. Nucleic Acids Res 2003, 3 I:3429-3431.

109. Hertel J, Lindemeyer M, Missal K, Fried C, Tanzer A, Flamm C, Hofacker IL, Stadler PF: The Students of Bioinformatics Computer Labs 2004 and 2005: The Expansion of the Metazoan MicroRNA Repertoire. BMC Genomics 2006, 7:15. 